\title{
A mystery unraveled: nontumorigenic pluripotent stem cells in human adult tissues
}

\author{
Ariel A Simerman ${ }^{1}$, Marcelo J Perone ${ }^{2}$, María L Gimeno², Daniel A Dumesic ${ }^{1}$, and Gregorio \\ D Chazenbalk, $\mathrm{PhD}^{\dagger}{ }^{\dagger}$ \\ ${ }^{1}$ The University of California, David Geffen School of Medicine, Department of Obstetrics and \\ Gynecology, 10833 Le Conte Ave, Box 951740, Los Angeles, CA 90095-1740, USA Fax: +1 310 \\ 2063670 \\ 2Instituto de Investigación en Biomedicina de Buenos Aires (IBioBA) - CONICET - Partner \\ Institute of the Max Planck Society, Buenos Aires, Argentina
}

\begin{abstract}
Introduction: Embryonic stem cells and induced pluripotent stem cells have emerged as the gold standard of pluripotent stem cells and the class of stem cell with the highest potential for contribution to regenerative and therapeutic application; however, their translational use is often impeded by teratoma formation, commonly associated with pluripotency. We discuss a population of nontumorigenic pluripotent stem cells, termed Multilineage Differentiating Stress Enduring (Muse) cells, which offer an innovative and exciting avenue of exploration for the potential treatment of various human diseases.
\end{abstract}

Areas covered: This review discusses the origin of Muse cells, describes in detail their various unique characteristics, and considers future avenues of their application and investigation with respect to what is currently known of adult pluripotent stem cells in scientific literature. We begin by defining cell potency, then discuss both mesenchymal and various reported populations of pluripotent stem cells, and finally delve into Muse cells and the characteristics that set them apart from their contemporaries.

Expert opinion: Muse cells derived from adipose tissue (Muse-AT) are efficiently, routinely and painlessly isolated from human lipoaspirate material, exhibit tripoblastic differentiation both spontaneously and under media-specific induction, and do not form teratomas. We describe qualities specific to Muse-AT cells and their potential impact on the field of regenerative medicine and cell therapy.

\section{Keywords}

human pluripotent stem cells; Muse cells; nontumorigenic; regenerative medicine

\footnotetext{
(C) 2014 Informa UK, Ltd.

${ }^{\dagger}$ Author for correspondence gchazenbalk@mednet.ucla.edu.

Declaration of interest

The authors were supported by an NIH U54 grant number HD071836 and the University of California, Los Angeles. The authors have no other relevant affiliations or financial involvement with any organization or entity with a financial interest in or financial conflict with the subject matter or materials discussed in the manuscript apart from those disclosed.
} 


\section{Introduction}

Since their discovery over a century ago by German scientist Ernst Haeckel, stem cells, or stammzellen as he coined them, have dominated both scientific conversations and resources without contest [1-3]. Alongside the discovery of their capacity for self-renewal, definitions of cell potency, the ability of a cell to differentiate into various cell types, soon arose to classify different types of stem cells and their ability to give rise to adult tissues of the three embryonic germ cell lineages. The 'unipotent' stem cell differentiates into one cell type; for instance, a muscle stem cell differentiating into a mature muscle cell [4]. The 'oligopotent' stem cell, which includes the hematopoietic stem cell, differentiates into few, but not all, cell types within a specific tissue [5]. For example, human breast stem cells are organ-specific adult stem cells and can differentiate into the cell types within the breast tissue, negating teratoma formation when injected into an animal model [6,7]. Unfortunately, these adult stem cells have been shown to give rise to breast carcinomas, supporting the stem cell theory of carcinogenesis [8]. The 'multipotent' stem cell differentiates into all cell types from a specific germ layer, which includes the increasingly popular mesenchymal stem cell (MSC) $[9,10]$. The 'pluripotent' stem cell, made famous by the fervently investigated embryonic stem (ES) cell [11,12] and the induced pluripotent stem cell (iPS) [13], is able to differentiate into cells of all three germ cell layers: mesodermal, endodermal and ectodermal. Stem cell pluripotency is widely determined utilizing the classical 'teratoma assay' as this is believed to provide the most concrete evidence of their capacity for tripoblastic differentiation. Recently, investigators have challenged this scientific cornerstone, arguing that not only is the teratoma assay not standardized, but there exist various alternatives, including the detection of classical markers of pluripotency and in vitro spontaneous and induced differentiation, to detect developmental potential [14]. Finally, the 'totipotent' stem cell, the most primitive stem cell most commonly known as the zygote, is capable of differentiation into embryonic and extra-embryonic cell types and gives rise to entire organisms $[15,16]$. Pluripotent stem cells have emerged as the variety of stem cells most worthy of investigation through their potential regenerative and therapeutic applications; however, they face a significant obstacle, which has thus precluded their translational use.

Pluripotent stem cells characteristically give rise to teratoma formation through their propensity for uncontrolled self-renewal and tripoblastic differentiation. Such has been the burden borne by investigators of ES and iPS cells in recent years, hindering the the therapeutic potential of these cells and quickly halting clinical trial. Recently, a population of pluripotent stem cells has been discovered with the potential to lift this encumbrance. Multilineage Differentiating Stress Enduring (Muse) cells were isolated under severe cellular stress conditions, from human bone marrow and dermal fibroblasts retaining selfrenewing properties despite their lack of teratoma formation when injected into immunedeficient mice [17]. Muse cells have since been isolated from human adipose tissue lipoaspirates [18], commercially available adipose stem cells (ASCs) [19] and goat fibroblasts [20]. Amongst a plethora of attractive qualities demonstrated by Muse cells, controlled proliferation in the absence of teratoma formation sets them apart from ES and iPS cells and offers an innovative and exciting avenue of exploration for the potential 
applications of these pluripotent stem cells to treat various human diseases. This review traces the origin of Muse cells and their emergence into the stem cell arena, detailing their various unique characteristics and future avenues of their application and investigation.

\section{Mesenchymal stem cells}

MSCs, of mesodermal lineage, are found in various mesenchymal tissues, including bone marrow, adipose and umbilical cord, and exert both trophic and anti-inflammatory effects in the event of tissue damage [21-26]. Despite their common consideration as a 'mulitpotent' stem cell population merely capable of differentiating into cell types of mesodermal origin, MSCs have been considered attractive and viable stem cell sources for regenerative medicine due to their accessibility, abundance and lack of tumorigenic propensity [27]. MSCs have been shown to differentiate across all three germ cell lineages with varying efficiencies both in vitro and in vivo, contributing to tissue regeneration and repair in some cases [28]. A total of 354 clinical trials have been conducted so far to investigate the potential application of autologous MSCs for stem cell therapy since 1995. Clinical trials for cancer, diabetes, multiple sclerosis, bone and cartilage disease, brain disease, heart disease, lung disease, graft versus host disease, liver disease, Crohn's disease, spinal cord injury and other disorders at various phases are in progress; however, safety issues and tumor formation in a few cases preclude wider clinical application [29].

\section{Embryonic and induced pluripotent stem cells: a shift in perspective}

In the early 1980s, ES cells derived from human blastocytes demonstrated the potential to revolutionize the field of regenerative medicine, and thereby, treat a exhaustive list of previously irreversible disorders through their capacity to generate tissues of all three germ lines $[11,12,30]$. Their unlimited proliferative power as well as their ability to remain in an undifferentiated state in cell culture conditions for $4-5$ months created a wave of intrigue that carried over into the mid 2000s. However, evidence soon emerged that ES cells produce immunorejection upon transplantation and form teratomas as a result of their unlimited proliferative capacity [31]. These hindrances to the translational use of ES cells along with debates surrounding the bioethical issues concerning the usage of human embryos essentially removed ES cells from the forefront of regenerative medicine.

Solving the ethical dilemmas surrounding the use of ES cells toward cell therapy, iPS cells became the new subject of interest in the stem cell field [13,32]. iPS cells have the capacity to reprogram, through an intricate mechanism involving the introduction of the so-called 'Yamanaka factors', including Nanog, Oct, Sox2, c-Myc and Klf4, which subsequently became the characteristic markers that establish pluripotency: the ability to self-renew and generate cells from the three germ lines and thus form teratomas [13,33-35]. Though iPS cells resolve the concerns of immunorejection because they can be generated from a patient's own, or autologous, cells. They seem also to obviate ethical issues that hinder the use of stem cells extracted from human embryos. Yet the production of teratomas upon transplantation as a result of unbridled cell proliferation, as well as an extremely low survival rate for both iPS and ES cells upon reintroduction to the host organism, impede the translational use of these cells [36,37]. Integration of viral transgenes into the host genome 
of iPS cells increases the risk of tumor formation and has led to the exploration of alternative avenues of programming, including the use of adenovirus vectors and plasmids $[38,39]$. Recently, efforts have been made to improve iPS cell generation, specifically to eliminate transgene integration and increase programming efficiency, including the induction of fewer transcriptional factors and direct delivery of reprogramming proteins [40,41]. Mature iPS cells have been found to possess that mature iPS cells possess an epigenetic memory, defined by the remnants of post-translational histone and DNA modifications, preventative of entirely successful reprogramming, often restricting their physiological function to that of a cell within the same lineage as the original stem cell source $[34,42,43]$. This phenomenon may be attributable to an organ-specific adult stem cell origin $[44,45]$. Despite momentous efforts invested in the study of both ES cells and iPS cells, little progress has come in resolving in resolving the defects that stand in the way of the use of these stem cells toward cell therapy in humans. Consequently, there exists a demand for a pluripotent stem cell population with a high post-transplantation survival rate that does not undergo teratogenesis in vivo.

\section{Adult pluripotent stem cells}

Various populations of adult pluripotent stem cells have been put forth as potential candidates for application in regenerative medicine, but have been subsequently scrutinized, and in most cases, outright challenged. In 2002, multipotent adult progenitor cells (MAPCs), derived from bone marrow, were reported to differentiate into all three embryonic germ cell layers both in vivo and in vitro without forming teratomas to contribute to chimeric offspring when injected into a mouse model and to regenerate tissue in the wake of myocardial infarction [46,47]. However, investigators have found it exceedingly difficult to replicate these data, thus putting to question the validity of these cells. Soon after the controversial advent of MAPCs, investigators isolated human marrow-isolated adult multilineage inducible cells, which too exhibited the potential for tripoblastic differentiation without teratoma formation [48]. However, it is unclear how MAPCs differ from other MSCs isolated from bone marrow [49]. Another population of adult pluripotent stem cells, termed very small embryonic-like stem cells (VSELs), met a similar fate to MAPCs soon after their advent. VSELs, isolated from bone marrow and umbilical cord blood, were also said to be pluripotent and not form teratomas [50]. However, the existence of a truly pluripotent population of VSELs was quickly refuted upon further investigation [51]. Unrestricted somatic stem cells (USSCs), derived from umbilical cord blood, emerged in 2011, boasting tripoblastic differentiation potential without the molecular backing of pluripotent stem cell marker expression [52]. As USSCs were shown to express epigenetic features of pluripotent stem cells and were able to maintain an uncommitted state, they have been proposed as a possible avenue for iPS cell generation as well as for application in regenerative therapy [52].

Most recently, it has been shown in mice that a method of $\mathrm{pH}$ reduction, termed stimulustriggered acquisition of pluripotency (STAP), indeed confers pluripotency to somatic cells by a not yet well understood reprogramming phenomenon. Obokata et al. showed that exposing splenic $\mathrm{CD} 45+$ lymphocytes to $\mathrm{pH}$ 5.7, followed by incubation with leukemia inhibitory factor, confers pluripotency and 'stemness' after 7 days in culture [53]. Despite 
the fact that STAP cells appear to demonstrate a limited capacity of self-renewal, they form teratomas, falling into a similar category as ES and iPS cells, and thus, impeding their clinical and translational value. Further investigation must be pursued in order to elucidate the mechanism behind the advent of these cells.

\section{The emergence and characterization of Muse cells}

The definition of 'pluripotency' relies upon a cell's ability to differentiate into the three embryonic germ layers [54]. When applied to stem cells in particular, this definition encompasses the capacity for self-renewal as well. In most cases, cell pluripotency is often both a blessing and a curse as the faculty for tripoblastic differentiation and self-renewal is frequently uncontrolled and often materializes in teratoma formation. Such is the case with ES and iPS cells, hindering the exploitation of their pluripotency for regenerative purposes.

In 2010, a research team at the Tohoku University in Sendai, Japan, successfully isolated a population of pluripotent MSCs from bone marrow aspirates and human skin fibroblasts through the induction of severe cellular stress, from bone marrow aspirates and human skin fibroblasts. Termed Muse cells, this cell population expresses the pluripotency marker stagespecific embryonic antigen-3 (SSEA-3) as well as the mesenchymal marker CD105 [17]. When cultured in suspension, Muse cells form cell clusters analogous to ES cell-derived embryoid bodies [17]. In isolating stem cells from human adipose tissue lipoaspirates, imposing alternate, yet increasingly severe stress conditions, our research team at the University of California, Los Angeles, discovered morphologically identical cell clusters that are recognized by pluripotent stem cell markers (unpublished data, Chazenbalk, 2008). Upon further investigation, these cells too proved to be the SSEA-3/CD105-positive Muse cells, distinguished as Muse cells derived from adipose tissue (Muse-AT) [18], which had previously been described in bone marrow aspirates and skin fibroblasts [17]. More recently, investigators have shown that Muse cells can be isolated from intact human adipose tissue and commercially available human ASCs through SSEA-3 cell sorting techniques [19]. Muse-AT cells have been subjected to extensive immunocytochemical analysis as well as microarray analysis to assess their morphological and functional properties. Similar to Muse cells derived from bone marrow aspirates and skin fibroblasts [17], Muse-AT cells grow in suspension as cell clusters reminiscent of ES cells and in addition to SSEA-3 express the classic pluripotency markers Sox2, Oct3/4, Nanog, TRA-1-60 and TRA-1-81 (Figure 1) $[17,18]$. Muse-AT cells, like Muse cells derived from bone marrow aspirates and skin fibroblasts, can differentiate into mesodermal, endodermal and ectodermal embryonic germ lineages spontaneously and with the use of tissue-specific culture media [17,18,55]. Immunocytochemistry studies showed that Muse-AT cells express markers for adipocytes, myocytes, hepatocytes and neural cells in both naive and induced Muse-AT cells, supporting both spontaneous and induced tripoblastic differentiation [18]. They have been shown to differentiate into cells of mesodermal, endodermal and ectodermal lineage spontaneously, with 23, 20 and 22\% respective efficiencies and under induction, with 82, 75 and $78 \%$ respective efficiencies, in vitro, exhibiting morphological characteristics of each lineage after a mere 3 days in culture. Muse-AT cells demonstrated formation of lipid droplets when induced to differentiate into adipocytes, characteristically fused nuclei and smooth muscle 
striations when induced to differentiate into myocytes, and long, finger-like projections, typical of neurons, when induced to differentiate into neural cells (Figure 1) [18].

\section{Nontumorigenicity of Muse cells}

Despite their delineation as pluripotent stem cells, Muse cells exhibit both low proliferative and telomerase activities, a normal karyotype as well as asymmetric growth, and thus, do not undergo tumorigenesis or teratoma formation when transplanted into a host organism [17,19]; unpublished data, Heneidi 2013) (Figure 2). While both the necessity and validity of the teratoma assay as an indicator of true pluripotency has been challenged in recent years, tumor formation has typically been considered to go hand-in-hand with both pluripotency and self-renewal [14,56-58]. Muse cells do not undergo unbridled proliferation or tumor formation, setting them apart from ES and iPS cells. When transplanted into the testes of immune-deficient mice, Muse cells did not form teratomas, while ES cells formed large teratomas within $8-10$ weeks [19]. As pluripotency and tumorigenesis have commonly been considered two sides of the same coin, this begs the question as to what factors allow Muse cells to avoid tumor formation while retaining their capacity for differentiation into all three germ lineages. Low telomerase activity, in part, is responsible for this scientific anomaly [19].

Muse cells exhibit a slight increase in expression of Sox2, Nanog, Oct3/4 as compared with non-muse cells and to ASCs (twofold to fourfold) [17,18]. Interestingly, Muse cells have much lower expression of the so-called 'Yamanaka factors' in comparison with iPS cells (> $10^{5}$ fold decrease) [55]. This intermediate expression of the genes that have been shown to confer pluripotency and teratogenesis may explain how Muse cells retain their lineage plasticity while simultaneously negating teratoma formation.

It is well established that Lin28, a RNA-binding protein gene, functions to maintain both pluripotency and tumorigenesis in ES and iPS cells. Let-7, a microRNA that regulates embryonic development, cell differentiation and tumor suppression, has the opposite effect [59]. While overexpression of Let-7 blocks Lin 28 gene expression, strong Lin 28 expression degrades Let-7, maintaining a 'yin yang' balance [59]. Initial studies found that Let-7 was a critical factor in the global decrease of microRNA, which has been observed in numerous cancers [59]. In contrast, ES and iPS cells have a very high Lin28/Let7 ratio, which is believed to lead these cells, in part, on a pathway to tumorigenic proliferation and, subsequently, cancer [59]. Interestingly, Muse cells retain their pluripotent capacity in the absence of a strong Lin28 influence (> $10^{5}$ fold decrease in comparison with iPS cells) [55]. Furthermore, Let-7 seems to be a critical master regulator of cell proliferation in preventing Muse-AT cells from forming teratomas (unpublished data, Chazenbalk, 2013). Overexpression of Let-7 in Muse cells could potentially play a critical role in inhibiting Lin28 expression, and therefore, would protect these cells from tumorigenic proliferation and teratoma formation after transplantation in vivo. Transgenic overexpression of Let-7 in Muse cells is a putative target for further exploration.

Cyclin-dependent kinase inhibitor 2A (CDKN2A), a key tumor suppressing factor, is another interesting gene found in Muse-AT cells that may provide insight into this intriguing 
association. CDKN2A is tightly linked with p16INK4a, which function together as a tumorsuppressing complex [60]. Repression of this complex promotes the isolation of cells in breast tissue with a high degree of lineage plasticity, low telomerase activity and the propensity for tumor formation [61]. Interestingly, CDKN2A is expressed in Lin28-negative Muse-AT cells, which reap the benefits of the pluripotency, nontumorigenicity and low telomerase activity. Upon further investigation, this genetic combination may illuminate the mechanism behind the pluripotent cell plasticity and tumor suppression exhibited by Muse cells.

\section{Awakening Muse cells from quiescence}

The application of stem cells in regenerative medicine has often been impeded by a low survival rate, typically $<3 \%$, when exposed to the high-stress engraftment environment [62-64]. The harsh milieu of the recipient site is exacerbated in instances of acute injury, which includes myocardial infarction, ischemic injury and stroke, hindering cell survival and, in some cases, inducing unwanted differentiation $[65,66]$. Previously, investigators have employed various tactics to acclimatize stem cells to pro-apoptotic factors, including hypoxia, malnutrition, pro-inflammatory cytokines, and reactive oxygen and nitrogen species, in order to enhance stem cell survival in vivo. Hypoxia preconditioning, a process in which stem cells are introduced to hypoxic conditions for $24-48 \mathrm{~h}$ prior to transplantation, has been shown to increase their likelihood of survival $[67,68]$. However, as the induction of a high-stress environment is imperative to Muse cell activation from their quiescent state, they are inherently conditioned to endure and thrive when transplanted in vivo [17,18]. Muse cells were most efficiently isolated from bone marrow aspirates and skin fibroblasts utilizing a long-term trypsin incubation, or proteolytic digestion, of the tissue [17]. Alternatively, subjecting human adipose tissue lipoaspirate material to even severe cellular stress (longterm exposure to the proteolytic enzyme collagenase, serum deprivation, low temperatures and hypoxia) yielded a highly purified population of Muse-AT cells [18]. The innate endurance of Muse cells to a high-stress environment supports their capacity for survival and the ultimate translational objective of tissue regeneration in vivo.

Muse cells, prior to cellular stress disruption, are present in a quiescent state under normal physiological circumstances within the cellular niche $[17,18]$. Multiple adult stem cell lineages have been shown to exist in a quiescent state at various time points throughout their lifespan, including hematopoietic stem cells and epithelial stem cells, which allegedly play a role in the preservation of their self-renewal [69]. Quiescence functions in the maintenance of malignancy in cancer stem cells and contributes to the susceptibility for cancer relapse notwithstanding cancer treatment [69]. Quiescent stem cells, including Muse-AT cells, are mobilized by the disruption of the cellular niche attributed to CXCL2, a critical chemokine involved in stem cell homing [70]. CXCL2, overexpressed in cancer cells, contributes both to cancer cell survival and malignancy. Studies show that preconditioning MSCs with CXCL2 increases post-transplantation survival rates after myocardial infarction [70]. CXCL2 is expressed 770 folds higher in Muse-AT cells as compared with ASCs and could therefore explain the inherent resistance of Muse-AT cells to cellular stress from a genetic perspective [18]. 
Our microarray data, showing up- and downregulation of 144 critical genes involved in cell death and survival (e.g., SGK1, MDH1, ATF2, HSPA8, PDIA3, BRD1, CALM1, NR4A2, GATA2, CDK6, NUF2, CDK6, BRC1, BUB1B and CCXL2), suggest that significant fluctuation in expression of these genes could be required to activate Muse cells from quiescence [18]. Furthermore, Muse-AT cells overexpress ALDH1A2 (47-fold change versus ASCs) and SOD2 (41-fold change versus ASCs), which boast antioxidative stress and antiapoptotic functions [70-72]. Interestingly, DNA repair genes are generally upregulated in Muse-AT cells, indicating a high capacity to resist DNA damage at the hands of cellular stress [18].

\section{Muse cells and tissue regeneration}

Green fluorescent protein (GFP)-labeled Muse cells injected intravenously into damaged skin, muscle and liver tissue of immune-deficient mice were able to differentiate accordingly in vivo and contribute vastly to tissue regeneration within $2-4$ weeks of engraftment [17]. Muse cells integrated into and formed dermis, gastrocnemius muscle and liver tissue (Figure 3) [17]. Furthermore, Muse cells were shown to treat fulminant hepatitis, muscle degeneration and skin injury in a mouse model (Figure 3). Integrated Muse cells were detected and tracked by lentivirus-GFP and human anti-Golgi complex immunoreactivity, human albumin and antitrypsin in the fulminant hepatitis model, human dystrophin in degenerated muscle and cytokeratin 14 in injured skin [17].

It has been shown that mature melanocytes derived from Muse cells have the capacity to contribute to tissue regeneration after grafting into the damaged skin of a mouse model [73]. Muse cells were utilized to create 3D cultured skin in vitro, which was then transplanted in vivo to the back skin of immune-deficient mice. Muse-derived melanocytes were pigmented, as compared with the negative control, and tested positive for human melanocyte markers (Figure 3). These studies suggest that Muse cells may be an ideal candidate population for autologous transplantation to treat melanocyte dysfunctions such as vitiligo.

\section{Muse cells and the immune system}

In adipose tissue, Muse-AT cells reside with both the adipocyte and the stromal vascular fractions, and presumably interact with both ASCs and adipose tissue macrophages (ATM) [18]. It has been shown that cross-talk between ASCs and ATM contributes to cell plasticity, the maintenance of quiescence, adipogenesis and ASC formation [74]. On the other hand, it is well known that adipose tissue is immunologically dynamic, in which there exist different types of leukocytes such as CD4+ and CD8+T cells, T regulatory (Treg) cells, macrophages and $\mathrm{B}$ lymphocytes, each of which play a versatile role in inflammation and immunometabolism [75]. Therefore, ASC and adipose immune cell interaction with neighboring Muse-AT cells may have implications for their lineage plasticity, adipose tissue differentiation, tissue repair and the production and recruitment of signaling molecules in times of cellular stress, acute damage and dysregulated metabolism [18].

The development of immunosuppressive drugs has made possible the treatment of several autoimmune disorders and cell and solid organ transplantation. However, chronic immunosuppression leads to detrimental effects, including susceptibility to infection, renal 
failure, diabetes, ulcers and risk of tumorigenesis [76]. Therefore, novel, effective and specific immunosuppressors without deleterious effects must be developed for clinical use. Numerous studies have demonstrated that MSCs possess immunosuppressive properties and modulate the function of a wide range of immune cell subpopulations of both the innate and the adaptive arms of the immune system, including $\mathrm{T}$ and $\mathrm{B}$ lymphocytes, natural killer and dendritic cells and induced Treg lymphocytes both in vivo and in vitro. This immunomodulatory role of MSCs has been attributed to both their capacity to synthesize and release soluble mediators and establish cell-to-cell contact with target cells. Several MSC-derived messengers have been identified as responsible for the mediation of immunomodulatory activity on $\mathrm{T}$ cell responses such as indoleamine 2,3-dioxygenase (IDO), prostaglandin E2, TGF- $\beta$, hepatocyte growth factor, TNF- $\alpha$ stimulated gene/protein 6 , and NO [77-82]. It is known that human MSCs inhibit $\mathrm{T}$ cell proliferation in vitro, a process that does not require major histocompatibility complex (MHC) restriction [83] but rather depends on the pro-inflammatory environment [84], where the IDO pathway seems to play a pivotal role [85] as well as HLA-G5 and IL-10 by cell contact [86]. Moreover, suppression of T lymphocyte proliferation exerted by MSCs also occurs through other mechanisms in which the cytokine microenvironment seems to play a decisive role. Importantly, if MSC-mediated immunosuppression does not require MHC restriction, one might speculate that it would be possible to use allogeneic MSCs to prolong the lifespan of transplanted organs or to treat autoimmune disorders.

ES cells have also shown a comparable ability to downregulate a T cell-mediated immune response via the arginase-dependent pathway [87]. However, the immunogenicity of ES cells and MSCs has been questioned as it has been demonstrated using noninvasive molecular imaging techniques that human ES cell xenotransplantation was rejected in immune-competent mice [88]. Therefore, it is clear to conclude that ES cells and MSCs are very efficient in modulating an immune response in vitro and in vivo. However, ES cell and MSC immunogenicity is a major concern and further studies must be performed to clarify this issue.

In this respect, we are currently planning experimental studies to address whether human Muse-AT cells have an immunomodulatory capacity as well as immunoprivileged features using both immune-deficient and immune-competent mice. As previously described, MuseAT cells overexpress key molecules responsible for cell homing and cell-to-cell connections between immune cells such as CXCL2 and intercellular adhesion molecule 1 (ICAM1) [18]. CXCL2, also called macrophage inflammatory protein 2-a, is implicated in neutrophil recruitment as an early step in controlling tissue injury and infections [89]. ICAM1, also known as CD54, a member of the immunoglobulin superfamily, is significantly increased upon cell activation, functions in signal transduction with pro-inflammatory effects, and is involved in inflammation and leukocyte extravasation. ICAM1 binds to macrophage adhesion ligand-(Mac)1, CD11a/CD18 (LFA-1) and CD11c/CD18 [90]. We have also found that Muse-AT cells exclusively express specific CD genes, as opposed to ASCs, which allow distinguishing both cell types based on their immunophenotype characteristics [18]. Furthermore, Muse-AT cells express three connexin genes GJA4, GJB2, GJB4, as well as C1orf71 (CNST) [18]. C1orf71 encodes the connexin recycling protein, Consortin, which 
mediates gap junction recycling and degradation [91]. Thus, additional studies are warranted to determine the role of these molecules on the surface of Muse-AT cells and their interaction with immune cells. The fundamental knowledge obtained by tackling these and other important questions should accelerate progress in the field and will ultimately lead to the development of novel immune therapeutics to control allogeneic cell and organ acceptance, ameliorate autoimmunity and improve metabolic complications.

\section{Muse cells as a source of iPS cells}

In addition to the notion that iPS cells are derived from organ-specific adult stem cells $[44,45]$, it has been reported that in adult human fibroblasts iPS cells are generated exclusively from Muse cells, supporting the elite model of iPS cell generation rather than the stochastic model [55]. The stochastic model posits that iPS cells can be generated from every cell type, with varying efficiencies, through the induction of the Yamanaka factors [13]. In support of the stochastic model, investigators have described the highly specific process through which cells are reprogrammed to iPS cells, detailing the molecular and genetic changes required for the induction of this process in any type of cell [92]. Conversely, the elite model conjectures that iPS cells can only be generated by a specific subset of cells [13]. When fibroblasts were subjected to transduction of the Yamanaka factors, only Muse cells underwent successful iPS cell generation [55]. Gene analysis shows that classical markers of tumorigenesis, including BCR1, CCMB1 and CCMB2, are highly expressed in iPS cells derived from Muse cells as compared with naive Muse cells [55]. In contrast, CDKN1A and CDKN2A, involved in tumor suppression, are highly expressed in Muse cells versus iPS cells derived from Muse cells [55]. This may shed light on the effects of the induction of the Yamanaka factors and their contribution to tumorigenesis and the propensity for teratoma formation inherent in iPS cells but not in Muse cells; however, further studies are required to elucidate this distinction.

\section{Muse cells in different species}

There currently exists a single report regarding the isolation of Muse cells from an animal model. Liu et al. isolated SSEA+ Muse cells from goat skin fibroblast cells (SFC) by FACS in order to investigate their application for somatic cell nuclear transfer (SCNT) [20]. They demonstrated that $2-4 \%$ of SFCs are SSEA+ Muse cells and that when applied as donor cells for SCNT, Muse cells contributed to a higher blastocyst rate than SSEA- SFCs in cloned embryos. Furthermore, blastocysts from donor Muse cells demonstrated enhanced quality as determined by total cell number and rate of apoptosis [20]. Confirming the existence of Muse cells in other mammalian species is a major step in the investigation of their potential and will undoubtedly inspire further animal studies to demonstrate their applicability for stem cell therapies.

\section{Future directions: Muse cells in regenerative medicine}

While investigators have adequately demonstrated the potential carried by Muse cells, it is now of the utmost importance to exploit this potential to explore various avenues of their translational application for regenerative medicine. As Muse-AT cells are so painlessly and efficiently isolated, one intriguing avenue is the formation of pluripotent stem cell banks for 
regenerative purposes. Harvesting and collecting Muse cells for the purpose of autologous stem cell therapies could prove useful for the regeneration of any type of tissue present in the human body and for the treatment of an exhaustive list of diseases, including neurological and immune disorders, and acute injuries to critical organs such as the heart and the brain. Furthermore, as a product of their undeniable genetic and physiological similarities, Muse cells have the potential to elucidate new avenues of cancer research, specifically with regards to cancer stem cells, quiescence, malignancy and post-treatment relapse [18,69]. Finally, as a feature of their high resistance to cellular stress, Muse cells could shed light on antiaging mechanisms and the rejuvenation of various tissues and organs facing age-related degeneration. It is commonly understood that the aging of human tissue coincides with an increase in oxidative stress damage to tissues as a consequence of an accumulation of free radicals and reactive oxygen species [93-96]. Moreover, DNA degradation and mutation contribute to the increasingly harsh milieu of the aging body. As Muse cells are inherently resistant to cellular stress, and genetically resilient to DNA damage, their application for the investigation of age-related and degenerative diseases is both relevant and promising.

Recently, pluripotent stem cells have been put forth as a promising tool for drug discovery, as they are said to model human physiology in a manner that may provide insight into the translational value of drugs in the early stages of screening [44,97,98]. It has been shown that various diseases can be successfully detected in differentiated adult pluripotent stem cells in vitro, providing a model for drug treatments [99]. iPS cells have been employed for this purpose; however, as their genetic reprogramming capacity remains under scrutiny, there exists a void that Muse cells have the potential to fill. Because Muse cells are 'natural pluripotent stem cells' present in every tissue of the body, they can be assessed at every stage of differentiation, from progenitor to terminally differentiated cell, allowing the full scope of a drug's cellular impact to be studied. Negating the induction of genetic manipulation, Muse cells are excellent candidates for use towards drug discovery.

Muse cells have emerged as a source of boundless investigation and innovation, revitalizing the conversation surrounding the application of pluripotent stem cells in regenerative medicine.

\section{Expert opinion}

Muse-AT cells, as compared with Muse cells isolated from alternate sources throughout the body, present themselves as the most promising stem cell source for application in regenerative medicine. Practically speaking, lipoaspirate material is easily accessible, abundant, and painlessly, routinely, and noninvasively extracted from the human body for both medical and cosmetic purposes. Previously, investigators have been hindered by a low yield of Muse cells from other sources, including dermal fibroblasts and bone marrow, as Muse cells make up only $1-3 \%$ of adult tissue. Hundreds of millions of adipose cells can be extracted from a mere $1-2$ liters of tissue, enhancing the number of extractable MuseAT cells. The isolation procedure, a 16-h digestion in a proteolytic enzyme under serum deprivation, low temperatures and severe hypoxia, is both time-efficient and cost-effective, 
negating the necessity for cell sorting techniques, which require expensive reagents and equipment.

As described above, Muse-AT cells posses an intrinsic predisposition for tripoblastic differentiation without teratoma formation, unlike pluripotent ES and iPS cells. Exposing Muse-AT cells to the same culture conditions used to induce ES and iPS differentiation into hepatocytes results in the formation of hepatocyte-like cells. Similarly, Muse-AT cells differentiate into neural cells in a comparable manner to ES and iPS cells. These results suggest that the potential of Muse-AT cells to treat disorders of the liver and brain parallel that of both ES and iPS cells; however, negating transgenic induction, they present zero teratogenic risk. Furthermore, Muse-AT cells exhibit medial to low expression of many genes involved in tissue development, cellular assembly and organization, cellular function and maintenance, DNA replication, repair and cell cycling, indicative of their intrinsic lack of tumorigenic susceptibility. As previously described, Muse-AT cells express genes associated with cell death and survival, as opposed to their ASC neighbors, indicating a genetic predisposition to the transition from the quiescent to the active state as a consequence of severe cellular stress.

According to microarray analysis performed on Muse-AT cells, they differentially express genes from the following functional groups in order of decreasing significance: cell death and survival, embryonic development, organismal development, tissue development, cellular assembly and organization and cellular function and maintenance (Figure 4). Many of these genes are highly conserved, with homologues present in numerous primordial organisms including yeast, Saccharomyces cerevisiae, Caenorhabditis elegans, Chlamydomonas, Trixis californica and Drosophila [18]. This suggests that Muse cells may function according to a highly conserved cellular mechanism related to cell survival in response to severe cellular stress, as well as the functional regeneration of damaged tissues and amputated limbs in primitive species $[66,100]$.

As Muse-AT cells have been thoroughly assessed for their pluripotency as well as their cellular stress resistance, and are so practically accessible, we believe them to be the strain of Muse cells most promising for the investigation of adult, nontumorigenic, pluripotent stem cells, and the eventual translation to regenerative stem cell therapy.

\section{Bibliography}

Papers of special note have been highlighted as either of interest $(\bullet)$ or of considerable interest $(\bullet)$ to readers.

1. Haeckel, E. Anthropogenie; oder entwickelungsgeschichte des menschen. 3rd. Engelmann, W., editor. W. Engelmann; Leipzig: 1877. p. 144

2. Brunt KR, Weisel RD, Li RK. Stem cells and regenerative medicine - future perspectives. Can J Physiol Pharmacol. 2012; 90(3):327-35. [PubMed: 22401558]

3. Maehle AH. Ambiguous cells: the emergence of the stem cell concept in the nineteenth and twentieth centuries. Notes Rec R Soc Lond. 2011; 65(4):359-78. [PubMed: 22332468]

4. Bentzinger CF, Wang YX, von Maltzahn J, Rudnicki MA. The emerging biology of muscle stem cells: implications for cell-based therapies. Bioessays. 2012; 35(3):231-41. [PubMed: 22886714] 
5. Majo F, Rochat A, Nicolas M, et al. Oligopotent stem cells are distributed throughout the mammalian ocular surface. Nature. 2008; 456(7219):250-4. [PubMed: 18830243]

6. Kao CY, Nomata K, Oakley CS, et al. Two types of normal human breast epithelial cells derived from reduction mammoplasty: phenotypic characterization and response to SV40 transfection. Carcinogenesis. 1995; 16(3):531-8. [PubMed: 7697810]

7. Trosko JE, Chang CC, Wilson MR, et al. Gap junctions and the regulation of cellular functions of stem cells during development and differentiation. Methods. 2000; 20(2):245-64. [PubMed: 10671317]

8. Tai MH, Chang CC, Kiupel M, et al. Oct4 expression in adult human stem cells: evidence in support of the stem cell theory of carcinogenesis. Carcinogenesis. 2005; 26(2):495-502. [PubMed: 15513931]

9. Kolf CM, Cho E, Tuan RS. Mesenchymal stromal cells. Biology of adult mesenchymal stem cells: regulation of niche, self-renewal and differentiation. Arthritis Res Ther. 2007; 9(1):204.

10. Uccelli A, Moretta L, Pistoia V. Mesenchymal stem cells in health and disease. Nat Rev Immunol. 2008; 8(9):726-36. [PubMed: 19172693]

11. Evans MJ, Kaufman MH. Establishment in culture of pluripotential cells from mouse embryos. Nature. 1981; 292(5819):154-6. [PubMed: 7242681]

12. Thomson JA, Itskovitz-Eldor J, Shapiro SS, et al. Embryonic stem cell lines derived from human blastocysts. Science. 1998; 282(5391):1145-7. [PubMed: 9804556]

13. Takahashi K, Yamanaka S. Induction of pluripotent stem cells from mouse embryonic and adult fibroblast cultures by defined factors. Cell. 2006; 126(4):663-76. [PubMed: 16904174]

14. Buta C, David R, Dressel R, et al. Reconsidering pluripotency tests: do we still need teratoma assays? Stem Cell Res. 2013; 11(1):552-62. [PubMed: 23611953]

15. Seydoux G, Braun RE. Pathway to totipotency: lessons from germ cells. Cell. 2006; 127(5):891904. [PubMed: 17129777]

16. Mitalipov S, Wolf D. Totipotency, pluripotency and nuclear reprogramming. Adv Biochem Eng Biotechnol. 2009; 114:185-99. [PubMed: 19343304]

17. Kuroda Y, Kitada M, Wakao S, et al. Unique multipotent cells in adult human mesenchymal cell populations. Proc Natl Acad Sci USA. 2010; 107(19):8639-43. [PubMed: 20421459]

18. $\bullet$ This is the first report of the isolation of Muse cells.

18. Heneidi S, Simerman AA, Keller E, et al. Awakened by cellular stress: isolation and characterization of a novel population of pluripotent stem cells derived from human adipose tissue. PLoS One. 2013; 8(6):e64752. [PubMed: 23755141]

20 . $\bullet$ This is the first report of the isolation of Muse cells from adipose tissue.

19. Ogura F, Wakao S, Kuroda Y, et al. Human adipose tissue possesses a unique population of pluripotent stem cells with non-tumorigenic and low telomerase activities: potential implications in regenerative medicine. Stem Cells Dev. 2014 Epub ahead of print.

20. Liu J, Yang Z, Qiu M, et al. Developmental potential of cloned goat embryos from an SSEA3(+) subpopulation of skin fibroblasts. Cell Reprogram. 2013; 15(2):159-65. [PubMed: 23441574]

21. Friedenstein AJ, Chailakhyan RK, Latsinik NV, et al. Stromal cells responsible for transferring the microenvironment of the hemopoietic tissues. Cloning in vitro and retransplantation in vivo. Transplantation. 1974; 17(4):331-40. [PubMed: 4150881]

22. Friedenstein AJ, Gorskaja JF, Kulagina NN. Fibroblast precursors in normal and irradiated mouse hematopoietic organs. Exp Hematol. 1976; 4(5):267-74. [PubMed: 976387]

23. Caplan AI. Mesenchymal stem cells. J Orthop Res. 1991; 9(5):641-50. [PubMed: 1870029]

24. Ye ZQ, Burkholder JK, Qiu P, et al. Establishment of an adherent cell feeder layer from human umbilical cord blood for support of long-term hematopoietic progenitor cell growth. Proc Natl Acad Sci USA. 1994; 91(25):12140-4. [PubMed: 7527553]

25. Zuk PA, Zhu M, Mizuno H, et al. Multilineage cells from human adipose tissue: implications for cell-based therapies. Tissue Eng. 2001; 7(2):211-28. [PubMed: 11304456]

26. Fang B, Song Y, Liao L, et al. Favorable response to human adipose tissue-derived mesenchymal stem cells in steroid-refractory acute graft-versus-host disease. Transplant Proc. 2007; 39(10): 3358-62. [PubMed: 18089385] 
27. Kuroda Y, Dezawa M. Mesenchymal stem cells and their subpopulation, pluripotent muse cells, in basic research and regenerative medicine. Anat Rec (Hoboken). 2013; 297(1):98-110. [PubMed: 24293378]

28. Prockop DJ. Marrow stromal cells as stem cells for nonhematopoietic tissues. Science. 1997; 276(5309):71-4. [PubMed: 9082988]

29. Wang S, Qu X, Zhao RC. Clinical applications of mesenchymal stem cells. J Hematol Oncol. 2012; 5:19. [PubMed: 22546280]

30. Martin GR. Isolation of a pluripotent cell line from early mouse embryos cultured in medium conditioned by teratocarcinoma stem cells. Proc Natl Acad Sci USA. 1981; 78(12):7634-8. [PubMed: 6950406]

31. Przyborski SA. Differentiation of human embryonic stem cells after transplantation in immunedeficient mice. Stem Cells. 2005; 23(9):1242-50. [PubMed: 16210408]

32. Maherali N, Sridharan R, Xie W, et al. Directly reprogrammed fibroblasts show global epigenetic remodeling and widespread tissue contribution. Cell Stem Cell. 2007; 1(1):55-70. [PubMed: 18371336]

33. Stadtfeld M, Hochedlinger K. Induced pluripotency: history, mechanisms, and applications. Genes Dev. 2010; 24(20):2239-63. [PubMed: 20952534]

34. Plath K, Lowry WE. Progress in understanding reprogramming to the induced pluripotent state. Nat Rev Genet. 2011; 12(4):253-65. [PubMed: 21415849]

35. Cunningham JJ, Ulbright TM, Pera MF, Looijenga LH. Lessons from human teratomas to guide development of safe stem cell therapies. Nat Biotechnol. 2012; 30(9):849-57. [PubMed: 22965062]

36. Geng YJ. Molecular mechanisms for cardiovascular stem cell apoptosis and growth in the hearts with atherosclerotic coronary disease and ischemic heart failure. Ann N Y Acad Sci. 2003; 1010:687-97. [PubMed: 15033813]

37. Fong CY, Gauthaman K, Bongso A. Teratomas from pluripotent stem cells: a clinical hurdle. J Cell Biochem. 2010; 111(4):769-81. [PubMed: 20665544]

38. Okita K, Yamanaka S. Induced pluripotent stem cells: opportunities and challenges. Philos Trans R Soc Lond B Biol Sci. 2011; 366(1575):2198-207. [PubMed: 21727125]

39. Fusaki N, Ban H, Nishiyama A, et al. Efficient induction of transgene-free human pluripotent stem cells using a vector based on Sendai virus, an RNA virus that does not integrate into the host genome. Proc Jpn Acad Ser B Phys Biol Sci. 2009; 85(8):348-62.

40. Kim D, Kim CH, Moon JI, et al. Generation of human induced pluripotent stem cells by direct delivery of reprogramming proteins. Cell Stem Cell. 2009; 4(6):472-6. [PubMed: 19481515]

41. Yu J, Hu K, Smuga-Otto K, et al. Human induced pluripotent stem cells free of vector and transgene sequences. Science. 2009; 324(5928):797-801. [PubMed: 19325077]

42. Hanna J, Saha K, Pando B, et al. Direct cell reprogramming is a stochastic process amenable to acceleration. Nature. 2009; 462(7273):595-601. [PubMed: 19898493]

43. Kim J, Woo AJ, Chu J, et al. A Myc network accounts for similarities between embryonic stem and cancer cell transcription programs. Cell. 2010; 143(2):313-24. [PubMed: 20946988]

44. Trosko JE. Commentary: "reprogramming or selecting adult stem cells?". Stem Cell Rev. 2008; 4(2):81-8. [PubMed: 18425605]

45. Trosko JE. Induction of iPS cells and of cancer stem cells: the stem cell or reprogramming hypothesis of cancer? Anat Rec (Hoboken). 2014; 297(1):161-73. [PubMed: 24293264]

46. Jiang Y, Jahagirdar BN, Reinhardt RL, et al. Pluripotency of mesenchymal stem cells derived from adult marrow. Nature. 2002; 418(6893):41-9. [PubMed: 12077603]

47. Dimomeletis I, Deindl E, Zaruba M, et al. Assessment of human MAPCs for stem cell transplantation and cardiac regeneration after myocardial infarction in SCID mice. Exp Hematol. 2010; 38(11):1105-14. [PubMed: 20621157]

48. D'Ippolito G, Diabira S, Howard GA, et al. Marrow-isolated adult multilineage inducible (MIAMI) cells, a unique population of postnatal young and old human cells with extensive expansion and differentiation potential. J Cell Sci. 2004; 117:2971-81. Pt 14. [PubMed: 15173316] 
49. Prockop DJ. Repair of tissues by adult stem/progenitor cells (MSCs): controversies, myths, and changing paradigms. Mol Ther. 2009; 17(6):939-46. [PubMed: 19337235]

50. Kucia M, Zuba-Surma EK, Wysoczynski M, et al. Adult marrow-derived very small embryoniclike stem cells and tissue engineering. Expert Opin Biol Ther. 2007; 7(10):1499-514. [PubMed: 17916043]

51. Miyanishi M, Mori Y, Seita J, et al. Do pluripotent stem cells exist in adult mice as very small embryonic stem cells? Stem Cell Rep. 2013; 1(2):198-208.

52. Santourlidis S, Wernet P, Ghanjati F, et al. Unrestricted somatic stem cells (USSC) from human umbilical cord blood display uncommitted epigenetic signatures of the major stem cell pluripotency genes. Stem Cell Res. 2011; 6(1):60-9. [PubMed: 20933485]

53. Obokata H, Wakayama T, Sasai Y, et al. Stimulus-triggered fate conversion of somatic cells into pluripotency. Nature. 2014; 505(7485):641-7. [PubMed: 24476887]

54. Wesselschmidt RL. The teratoma assay: an in vivo assessment of pluripotency. Methods Mol Biol. 2011; 767:231-41. [PubMed: 21822879]

55. Wakao S, Kitada M, Kuroda Y, et al. Multilineage-differentiating stress-enduring (Muse) cells are a primary source of induced pluripotent stem cells in human fibroblasts. Proc Natl Acad Sci USA. 2011; 108(24):9875-80. [PubMed: 21628574]

56. Ben-David U, Benvenisty N. The tumorigenicity of human embryonic and induced pluripotent stem cells. Nat Rev Cancer. 2011; 11(4):268-77. [PubMed: 21390058]

57. Gutierrez-Aranda I, Ramos-Mejia V, Bueno C, et al. Human induced pluripotent stem cells develop teratoma more efficiently and faster than human embryonic stem cells regardless the site of injection. Stem Cells. 2010; 28(9):1568-70. [PubMed: 20641038]

58. Lee AS, Tang C, Rao MS, et al. Tumorigenicity as a clinical hurdle for pluripotent stem cell therapies. Nat Med. 2013; 19(8):998-1004. [PubMed: 23921754]

59. Thornton JE, Gregory RI. How does Lin28 let-7 control development and disease? Trends Cell Biol. 2012; 22(9):474-82. [PubMed: 22784697]

62. This genetic balance may play a critical role in maintaining Muse cell pluripotency while deterring teratoma formation.

60. Reynolds PA, Sigaroudinia M, Zardo G, et al. Tumor suppressor p16INK4A regulates polycombmediated DNA hypermethylation in human mammary epithelial cells. J Biol Chem. 2006; 281(34):24790-802. [PubMed: 16766534]

61. Roy S, Gascard P, Dumont N, et al. Rare somatic cells from human breast tissue exhibit extensive lineage plasticity. Proc Natl Acad Sci USA. 2012; 110(12):4598-603. [PubMed: 23487770]

62. Hodgetts SI, Beilharz MW, Scalzo AA, Grounds MD. Why do cultured transplanted myoblasts die in vivo? DNA quantification shows enhanced survival of donor male myoblasts in host mice depleted of CD4+ and CD8+ cells or Nk1.1+ cells. Cell Transplant. 2000; 9(4):489-502. [PubMed: 11038066]

63. Oh JS, Kim KN, An SS, et al. Cotransplantation of mouse neural stem cells (mNSCs) with adipose tissue-derived mesenchymal stem cells improves mNSC survival in a rat spinal cord injury model. Cell Transplant. 2010; 20(6):837-49. [PubMed: 21054952]

64. Hofmann M, Wollert KC, Meyer GP, et al. Monitoring of bone marrow cell homing into the infarcted human myocardium. Circulation. 2005; 111(17):2198-202. [PubMed: 15851598]

65. Dayem AA, Choi HY, Kim JH, Cho SG. Role of oxidative stress in stem, cancer, and cancer stem cells. Cancers (Basel). 2010; 2(2):859-84. [PubMed: 24281098]

66. Kultz D. Molecular and evolutionary basis of the cellular stress response. Annu Rev Physiol. 2005; 67:225-57. [PubMed: 15709958]

70. - This article provides the foundation upon which the story of the stress response intrinsic to Muse cells has grown and become of great importance in determining their capacity for survival in vitro.

67. Chacko SM, Ahmed S, Selvendiran K, et al. Hypoxic preconditioning induces the expression of prosurvival and proangiogenic markers in mesenchymal stem cells. Am J Physiol Cell Physiol. 2010; 299(6):C1562-70. [PubMed: 20861473]

68. Abdollahi H, Harris LJ, Zhang P, et al. The role of hypoxia in stem cell differentiation and therapeutics. J Surg Res. 2009; 165(1):112-17. [PubMed: 20080246] 
69. Li L, Bhatia R. Stem cell quiescence. Clin Cancer Res. 2011; 17(15):4936-41. [PubMed: 21593194]

74. - The quiescent nature of Muse cells, prior to cellular stress disruption, further supports their resilience to harsh transplantation environments.

70. Huang J, Zhang Z, Guo J, et al. Genetic modification of mesenchymal stem cells overexpressing CCR1 increases cell viability, migration, engraftment, and capillary density in the injured myocardium. Circ Res. 2010; 106(11):1753-62. [PubMed: 20378860]

71. Vasiliou V, Nebert DW. Analysis and update of the human aldehyde dehydrogenase (ALDH) gene family. Hum Genomics. 2005; 2(2):138-43. [PubMed: 16004729]

72. Guo G, Yan-Sanders Y, Lyn-Cook BD, et al. Manganese superoxide dismutase-mediated gene expression in radiation-induced adaptive responses. Mol Cell Biol. 2003; 23(7):2362-78. [PubMed: 12640121]

73. Tsuchiyama K, Wakao S, Kuroda Y, et al. Functional melanocytes are readily reprogrammable from multilineage-differentiating stress-enduring (muse) cells, distinct stem cells in human fibroblasts. J Invest Dermatol. 2013; 133(10):2425-35. [PubMed: 23563197]

74. Chazenbalk G, Bertolotto C, Heneidi S, et al. Novel pathway of adipogenesis through cross-talk between adipose tissue macrophages, adipose stem cells and adipocytes: evidence of cell plasticity. PLoS One. 2011; 6(3):e17834. [PubMed: 21483855]

75. Winer S, Chan Y, Paltser G, et al. Normalization of obesity-associated insulin resistance through immunotherapy. Nat Med. 2009; 15(8):921-9. [PubMed: 19633657]

76. Engels EA, Pfeiffer RM, Fraumeni JF Jr, et al. Spectrum of cancer risk among US solid organ transplant recipients. Jama. 2011; 306(17):1891-901. [PubMed: 22045767]

77. Meisel R, Zibert A, Laryea M, et al. Human bone marrow stromal cells inhibit allogeneic T-cell responses by indoleamine 2,3-dioxygenase-mediated tryptophan degradation. Blood. 2004; 103(12):4619-21. [PubMed: 15001472]

78. Aggarwal S, Pittenger MF. Human mesenchymal stem cells modulate allogeneic immune cell responses. Blood. 2005; 105(4):1815-22. [PubMed: 15494428]

79. Ylostalo JH, Bartosh TJ, Coble K, Prockop DJ. Human mesenchymal stem/stromal cells cultured as spheroids are self-activated to produce prostaglandin E2 that directs stimulated macrophages into an anti-inflammatory phenotype. Stem Cells. 2012; 30(10):2283-96. [PubMed: 22865689]

80. Di, Nicola M.; Carlo-Stella, C.; Magni, M., et al. Human bone marrow stromal cells suppress Tlymphocyte proliferation induced by cellular or nonspecific mitogenic stimuli. Blood. 2002; 99(10):3838-43. [PubMed: 11986244]

81. Roddy GW, Oh JY, Lee RH, et al. Action at a distance: systemically administered adult stem/ progenitor cells (MSCs) reduce inflammatory damage to the cornea without engraftment and primarily by secretion of TNF-alpha stimulated gene/protein 6. Stem Cells. 2011; 29(10):1572-9. [PubMed: 21837654]

82. Sato K, Ozaki K, Oh I, et al. Nitric oxide plays a critical role in suppression of T-cell proliferation by mesenchymal stem cells. Blood. 2007; 109(1):228-34. [PubMed: 16985180]

83. Le, Blanc K.; Frassoni, F.; Ball, L., et al. Mesenchymal stem cells for treatment of steroid-resistant, severe, acute graft-versus-host disease: a phase II study. Lancet. 2008; 371(9624):1579-86. [PubMed: 18468541]

84. Comoli P, Ginevri F, Maccario R, et al. Human mesenchymal stem cells inhibit antibody production induced in vitro by allostimulation. Nephrol Dial Transplant. 2008; 23(4):1196-202. [PubMed: 18029377]

85. Ryan JM, Barry F, Murphy JM, Mahon BP. Interferon-gamma does not break, but promotes the immunosuppressive capacity of adult human mesenchymal stem cells. Clin Exp Immunol. 2007; 149(2):353-63. [PubMed: 17521318]

86. Selmani Z, Naji A, Zidi I, et al. Human leukocyte antigen-G5 secretion by human mesenchymal stem cells is required to suppress T lymphocyte and natural killer function and to induce CD4 +CD25highFOXP3+ regulatory T cells. Stem Cells. 2008; 26(1):212-22. [PubMed: 17932417]

87. Yachimovich-Cohen N, Even-Ram S, Shufaro Y, et al. Human embryonic stem cells suppress T cell responses via arginase I-dependent mechanism. J Immunol. 2010; 184(3):1300-8. [PubMed: 20032293] 
88. Swijnenburg RJ, Schrepfer S, Govaert JA, et al. Immunosuppressive therapy mitigates immunological rejection of human embryonic stem cell xenografts. Proc Natl Acad Sci USA. 2008; 105(35):12991-6. [PubMed: 18728188]

89. De, Filippo K.; Dudeck, A.; Hasenberg, M., et al. Mast cell and macrophage chemokines CXCL1/ CXCL2 control the early stage of neutrophil recruitment during tissue inflammation. Blood. 2013; 121(24):4930-7. [PubMed: 23645836]

90. Boscacci RT, Pfeiffer F, Gollmer K, et al. Comprehensive analysis of lymph node stromaexpressed Ig superfamily members reveals redundant and nonredundant roles for ICAM-1, ICAM-2, and VCAM-1 in lymphocyte homing. Blood. 2010; 116(6):915-25. [PubMed: 20395417]

91. del, Castillo FJ.; Cohen-Salmon, M.; Charollais, A., et al. Consortin, a trans-Golgi network cargo receptor for the plasma membrane targeting and recycling of connexins. Hum Mol Genet. 2010; 19(2):262-75. [PubMed: 19864490]

92. O’Malley J, Skylaki S, Iwabuchi KA, et al. High-resolution analysis with novel cell-surface markers identifies routes to iPS cells. Nature. 2013; 499(7456):88-91. [PubMed: 23728301]

93. Harman D. Aging: a theory based on free radical and radiation chemistry. J Gerontol. 1956; 11(3): 298-300. [PubMed: 13332224]

94. Harman D. The biologic clock: the mitochondria? J Am Geriatr Soc. 1972; 20(4):145-7. [PubMed: 5016631]

95. Hekimi S, Lapointe J, Wen Y. Taking a "good" look at free radicals in the aging process. Trends Cell Biol. 2011; 21(10):569-76. [PubMed: 21824781]

96. Wang $\mathrm{CH}, \mathrm{Wu} \mathrm{SB}, \mathrm{Wu}$ YT, Wei YH. Oxidative stress response elicited by mitochondrial dysfunction: implication in the pathophysiology of aging. Exp Biol Med (Maywood). 2013; 238(5):450-60. [PubMed: 23856898]

97. Kang KS, Trosko JE. Stem cells in toxicology: fundamental biology and practical considerations. Toxicol Sci. 2011; 120(Suppl 1):S269-89. [PubMed: 21163910]

98. Engle SJ, Puppala D. Integrating human pluripotent stem cells into drug development. Cell Stem Cell. 2013; 12(6):669-77. [PubMed: 23746976]

99. Yazawa M, Hsueh B, Jia X, et al. Using induced pluripotent stem cells to investigate cardiac phenotypes in Timothy syndrome. Nature. 2011; 471(7337):230-4. [PubMed: 21307850]

100. Meier P, Finch A, Evan G. Apoptosis in development. Nature. 2000; 407(6805):796-801. [PubMed: 11048731] 


\section{Article highlights}

- Pluripotent stem cells have the potential to revolutionize regenerative therapy

- The application of many pluripotent stem cells, such as embryonic and induced pluripotent stem cells, for treatment in humans is impeded by characteristic teratoma formation.

- Multilineage Differentiating Stress Enduring (Muse) cells exhibit pluripotent characteristics, including controlled self-renewal and tripoblastic differentiation.

- Muse cells do not form teratomas when transplanted in vivo.

- Muse cells derived from adipose tissue are the most efficiently isolated and applicable form of Muse cells in regenerative medicine. 

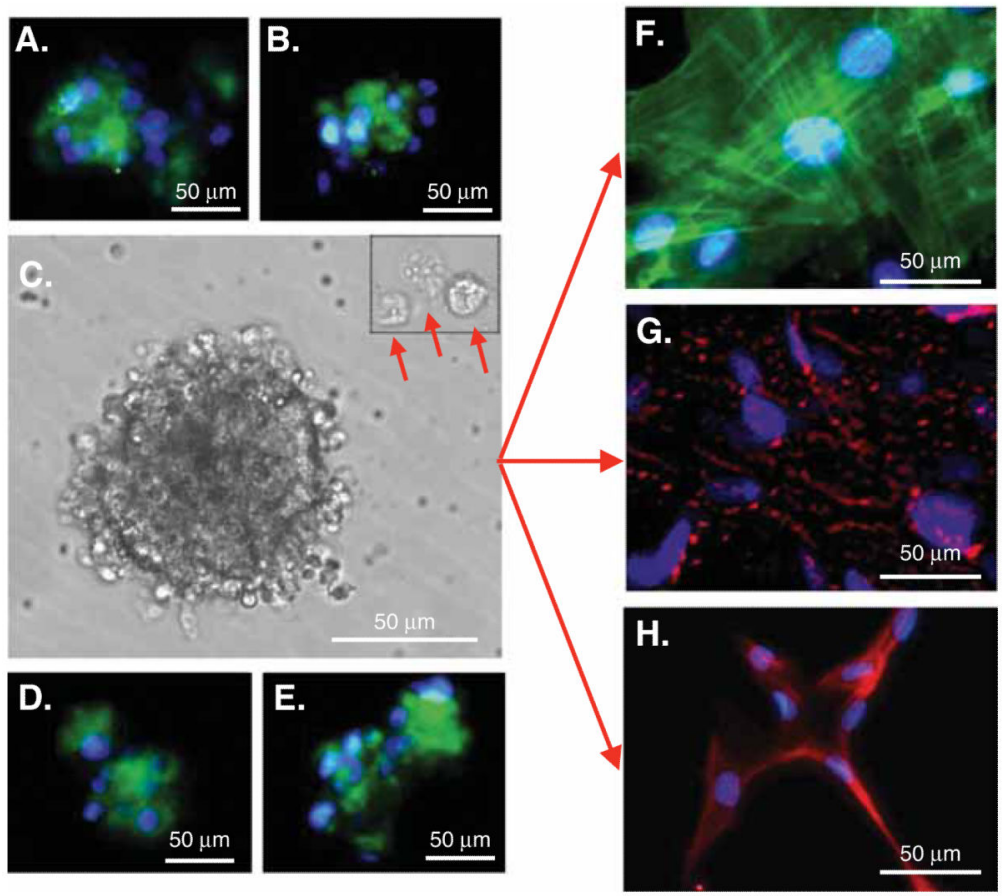

Figure 1. Multilineage Differentiating Stress Enduring (Muse) cell characterization by pluripotency markers and morphology

Immunostaining indicates that Muse cells express the pluripotency markers: (A) stagespecific embryonic antigen-3, (B) OCT3/4, (D) SOX2 and (E) Nanog. (C) Muse cells derived from adipose tissue (Muse-AT) cells grow in suspension, forming cell clusters as well as individual cells (red arrows). (F) Muse-AT cells were grown as adherent cells in the presence of myocyte differentiation medium. Formation of myocytes was detected using an antihuman MSA antibody. Nuclei were stained with DAPI (blue). (G) Muse-AT cells were grown as adherent cells in the presence of hepatocyte differentiation medium. Formation of hepatocytes was detected using an antihuman a-fetoprotein antibody. Nuclei were stained with DAPI (blue). (H) Isolated Muse-AT cells were grown for 7 days as nonadherent cells and then cultured for an additional 7 days as adherent cells. Neural-like cells were detected by immunofluorescence using an antihuman MAP2 antibody. Nuclei were stained with DAPI (blue). (Original magnification was $600 \times$ for figures $\mathbf{F}-\mathbf{H}$ ).

Reproduced from [18]. 

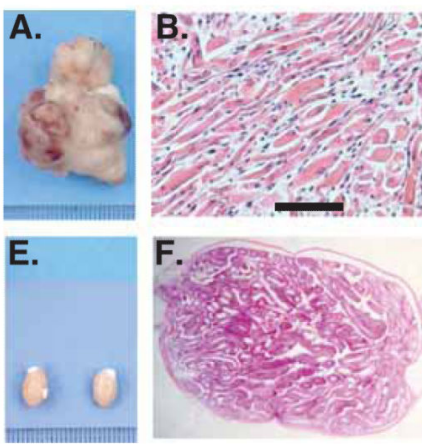

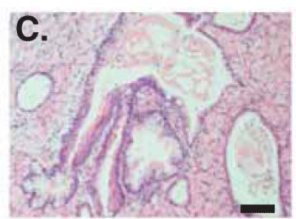

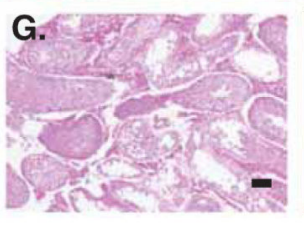
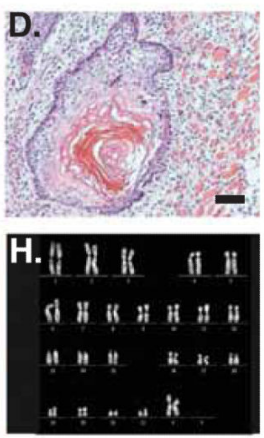

Figure 2. Nontumorigenicity of Multilineage Differentiating Stress Enduring (Muse) cells (A) Embryonic stem cells infused into immunodeficient mice (SCID mice) testes, formed teratomas within $8-12$ weeks. Histological analysis showed that the teratoma contained (B) muscle tissue, $(\mathbf{C})$ intestine-like structure and (D) keratinized skin. (E) Muse celltransplanted testes did not generate teratomas similar to untreated testes. (F,G) Testis injected with Muse cells maintained normal structure even 6 months postinjection. (H) Muse cells derived from adipose tissue cells showed normal karyotype. Scale bars $=100 \mu \mathrm{m}$. Reproduced from [17]. 

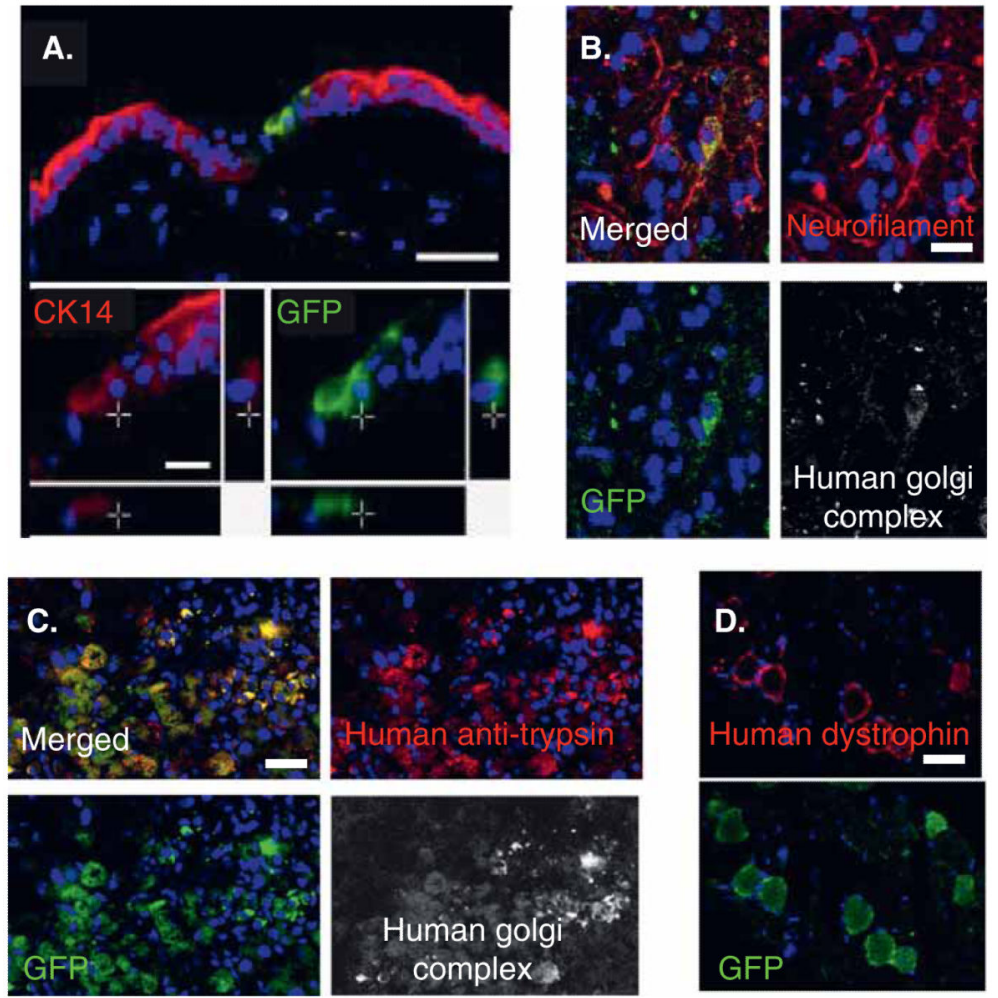

Figure 3. Multilineage Differentiating Stress Enduring (Muse) cells integrated into damaged tissue

GFP-positive human Muse cells integrated into a (A) skin injury model, (B) spinal cord injury model, (C) fulminant hepatitis model, and (D) a degenerated muscle model. GFPMuse cells were positive for (A) cytokeratin 14, (B) neurofilament albumin, (C) trypsin and (D) dystrophin. Muse cells were also positive for the human cell marker, antihuman Golgi complex, confirming that the positive cells were of human origin. Scale bars $=50 \mu \mathrm{m}(\mathrm{A}, \mathrm{B})$ and $100 \mu \mathrm{m}(\mathbf{C}, \mathbf{D})$.

GFP: Green fluorescent protein.

Reproduced from [17]. 


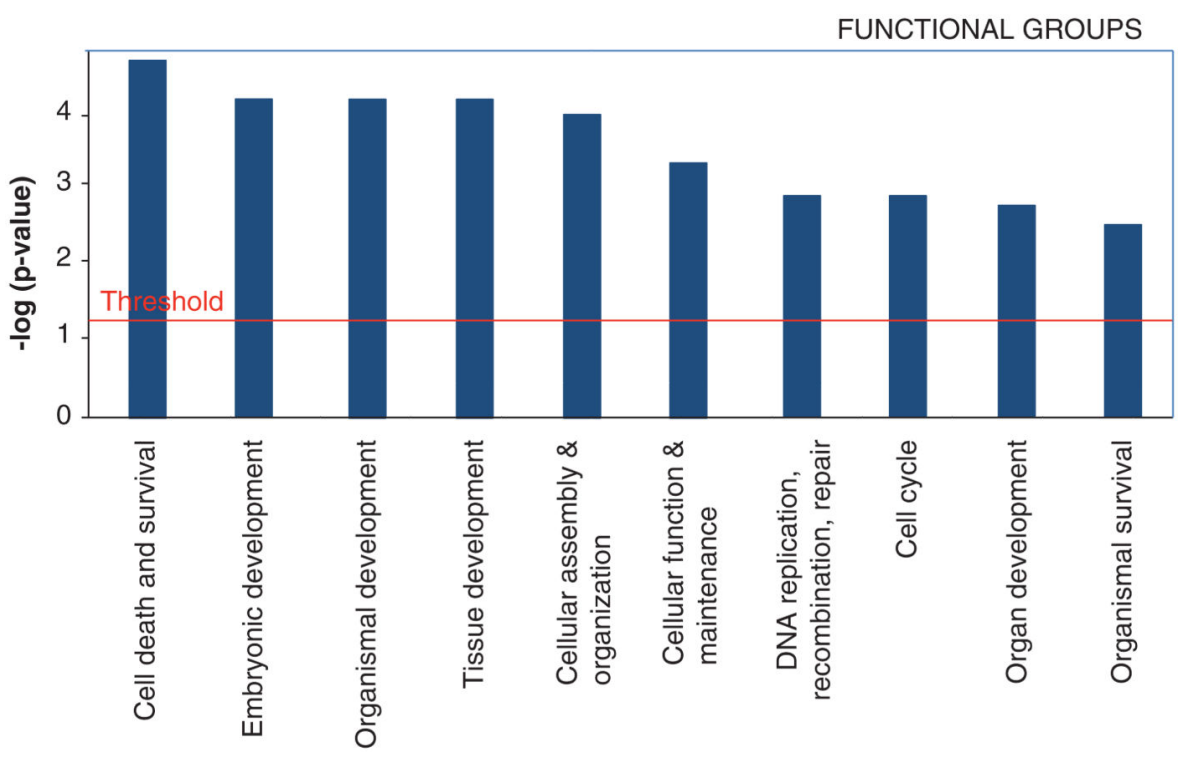

Figure 4. Identification of the top 10 functional groups of all differentially expressed genes (twofold or higher) in Muse cells derived from adipose tissue (Muse-AT) cells versus adipose stem cells (ASCs)

Fischer's exact test was used to calculate a $p$-value determining the probability of the association between the genes in the data set with functional groups and canonical pathways. Functional groups are displayed along the $x$-axis, while the $y$-axis displays logarithm of $p$ values calculated by Fisher exact between the ratio of the number of genes differentially expressed genes (twofold or higher) in Muse-AT versus ASCs in a given functional group or pathway divided by total number of genes that make up that functional group or pathway with a threshold for statistical significance set at 0.05 . The analysis was performed by Ingenuity Pathways analysis software.

Reproduced from [18]. 